\title{
HGF/MET in osteogenic differentiation of primary human palatal periosteum-derived mesenchymal stem cells
}

\author{
Noel Ye Naung ${ }^{1,2)}$, Warwick J Duncan ${ }^{1)}$, Rohana K. De Silva ${ }^{1)}$, and Dawn E. Coates ${ }^{1)}$ \\ Sir John Walsh Research Institute, Faculty of Dentistry, University of Otago, Dunedin, New Zealand \\ ${ }^{2}$ Pun Hlaing Hospitals, Yangon, Myanmar
}

(Received April 7, 2021; Accepted August 10, 2021)

\begin{abstract}
Purpose: This study aimed to determine expressions of hepatocyte growth factor (HGF) and MET proto-oncogene receptor tyrosine kinase (MET) in palatal periosteum (PP) and to examine the effect of HGF/MET on osteogenic differentiation of human palatal periosteum-derived mesenchymal stem cells (PD-MSCs).

Methods: HGF/MET proteins in human palatal periosteum $(n=3)$ were localized using immunohistochemistry. PD-MSCs $(n=3)$ were cultured in serum-free Essential 8 (E8) medium or osteogenic medium with and without Capmatinib, a selective ATP-inhibitor of MET. HGF concentration in vitro was measured with ELISA. Relative gene expression was quantified from PD-MSCs by quantitative reverse transcription real-time polymerase chain reaction.

Results: Immunohistochemistry detected co-localization of HGF and MET protein in PP. HGF protein levels were significantly higher $(P<0.05)$ in osteogenic media (day 21: $12.19 \pm 8.36 \mathrm{ng} / \mathrm{mL}$ ) than in E8 medium (day 21: $0.42 \pm 0.72 \mathrm{ng} / \mathrm{mL}$ ). MET inhibitor had a limited feedback effect on the expression profile of the osteogenic genes tested. Gene expression levels for all but three genes were comparable in serum-free and osteogenic media at all time points.

Conclusion: HGF/MET present in human PP and HGF is upregulated in vitro during osteogenesis; however the targeted pathways controlled by MET may not involve osteoblast maturation.
\end{abstract}

Keywords; hepatocyte growth factor, mesenchymal stem cells, MET, osteogenesis, periosteum

\section{Introduction}

Osteogenesis is a highly orchestrated process controlled by numerous signaling molecules, growth factors, and cytokines. It involves recruitment of mesenchymal stem cells (MSCs) and their induction to become osteoblasts that then produce mineralized bone matrix.

Hepatocyte growth factor (HGF), also called scatter factor, was discovered as a potent mitogen for mature hepatocytes and is secreted by MSCs [1]. It has important roles in organogenesis, tissue repair, and wound healing [2]. MET proto-oncogene receptor tyrosine kinase (MET), a member of the receptor tyrosine kinase family, is the only known receptor for HGF [3]. The MET receptor is also expressed by MSCs, and its activation is critical to embryogenesis and organogenesis [4].

Accumulating evidence indicates that HGF and MET have crucial roles in regulating osteogenesis [5]. Interestingly, one study noted that blocking MET signaling enhanced osteoblast differentiation and bone regeneration [6]. In addition, mutations in the MET receptor were associated with osteofibrous dysplasia, a congenital disorder affecting bone [7]. Similarly, sequential changes in HGF concentration were found to control MSC proliferation and osteogenic regeneration [5]. These findings highlight the

Correspondence to Dr. Dawn E. Coates, Sir John Walsh Research Institute, Faculty of Dentistry, University of Otago, 310 Great King Street, Dunedin 9016, Dunedin, New Zealand

E-mail: dawn.coates@otago.ac.nz

Color figures can be viewed in the online issue at J-STAGE.

doi.org/10.2334/josnusd.21-0164

DN/JST.JSTAGE/josnusd/21-016 importance of the HGF/MET signaling pathway on osteogenesis but raise questions regarding its role.

Because of safety concerns associated with serum-containing media, serum- and xeno-free media are increasingly used to expand stem cells [8]. Essential 8 (E8) medium is a defined serum- and xeno-free medium that has proven useful for growing and maintaining MSCs derived from palatal periosteum-derived tissue [9]. Several studies have reported that use of serum versus serum-free culture systems had a significant effect on HGF production and that this was subsequently associated with changes in the osteogenic capacity of MSCs. Ohshima et al. (2002) found that fetal bovine serum (FBS) stimulated HGF secretion by periodontal ligament and gingival fibroblasts [10]. In addition, Jun et al. (2007) reported elevated levels of HGF and abnormal cell proliferation and scattering when retinal pigment epithelial cells were cultured under serum-starved conditions [11].

To the best of the authors' knowledge, no previous study has investigated HGF production by human palatal periosteum-derived mesenchymal stem cells (PD-MSCs) cultured in serum- and xeno-free E8 medium. In addition, production of HGF by PD-MSCs during osteogenesis with or without blockage of MET has not been reported. This study aimed to localize HGF/MET in vivo and determine the concentration of HGF produced by PD-MSCs during osteogenesis and the relative expression of HGF/MET and osteogenic-related genes, with and without blockage of MET, by using the potent, highly selective MET inhibitor Capmatinib (INCB28060) [12].

\section{Materials and Methods}

\section{Participants}

Palatal periosteum was collected after obtaining written consent from patients and approval from the University of Otago Ethics committee (approval number: H13/104). Tissue was collected from three healthy patients (age 13, 14, and 20 years; one male, two females) undergoing open surgical exposure of impacted maxillary canines at the Faculty of Dentistry, University of Otago, New Zealand.

\section{Experimental design}

Full-thickness palatal periosteum (PP) tissue for immunohistochemistry was fixed in $10 \%$ neutral buffered formalin for $24 \mathrm{~h}$ at $4^{\circ} \mathrm{C}$ before being embedded in paraffin. Tissue for isolation of PD-MSCs was collected from the two-thirds of the PP closest to the bone. The top third containing the palatal epithelium was removed and discarded, and the accuracy of this process was validated with histological techniques. PP tissue was minced and grown as explant cultures in serum- and xeno-free E8 medium on vitronectin-coated plates (Thermo Fisher Scientific, Waltham, MA, USA).

\section{Immunohistochemical localization of HGF and MET proteins}

Paraffin-embedded serial sessions of PP tissue (thickness, $4 \mu \mathrm{m} ; n=3$ ) were deparaffinized, and antigen retrieval was performed with a heatinduced epitope retrieval method by boiling the slides in citrate buffer $(\mathrm{pH}$ 6.0) at $110^{\circ} \mathrm{C}$ for $10 \mathrm{~min}$ in a NxGen decloaking chamber (Biocare Medical, Pacheco, CA, USA).

Blocking was then conducted with $20 \%$ goat serum in $1 \%$ bovine serum albumin (BSA)/PBS, and slides were then incubated with a mouse anti-human HGF antibody $(10 \mu \mathrm{g} / \mathrm{mL}$; Abcam, Cambridge, UK) or rabbit anti-human MET antibody $(5 \mu \mathrm{g} / \mathrm{mL}$; Abcam). Isotype-matched negative controls of anti-mouse IgG (10 $\mu \mathrm{g} / \mathrm{mL}$; Santa Cruz Biotechnology, Dallas, TX, USA $)$ or anti-rabbit IgG $(5 \mu \mathrm{g} / \mathrm{mL}$; Dako, Glostrup, Denmark) were 
performed. All antibodies were diluted in 5\% goat serum with $1 \%$ BSA/ PBS and incubated overnight at $4^{\circ} \mathrm{C}$.

The sections were washed and then incubated with a biotinylated secondary antibody for $1 \mathrm{~h}$ at room temperature. Endogenous peroxidase activity was blocked with $0.3 \% \mathrm{H}_{2} \mathrm{O}_{2}$ prepared in methanol, and the samples were incubated with Vectastain $\mathrm{ABC}$ reagent (Vector laboratories, Burlingame, CA, USA), which was then detected with 3,3'-diaminobenzidine Chromogen (Sigma-Aldrich, St Louis, MO, USA). The slides were stained with hematoxylin and mounted with DPX before being scanned with an Aperio Digital Slide Scanning System (Leica Biosystems, Buffalo Grove, IL, USA).

\section{HGF protein production by PP cells in vitro}

Each cell line ( $n=3$; biological replicates) was cultured in triplicate wells for each of the three different conditions investigated. Phenotyping of these PP cells in E8 medium with antibodies to CD105, CD90, and CD73 via fluorescent-activated cell sorting (FACS) identified the presence of $73 \%$ triple-positive cells capable of trilineage differentiation, suggesting high numbers of MSCs. Trilineage differentiation of the cell lines revealed that they became adipocytes with Oil Red O-stained droplets, chondrocytes with Alcian blue-positive micromass formation, and osteoblasts with von Kossa-positive staining and nodules that stained Alizarin Red S-positive and produced osteocalcin [9]. Cells were seeded at a density of 8000 cells/ $\mathrm{cm}^{2}$ onto vitronectin-coated 6-well plates and cultured with E8 medium (using the method supplied with the E8 medium; Thermo Fisher Scientific). All media preparations were supplemented with $1 \%$ Antibiotic-Antimycotic (Thermo Fisher Scientific) and $10 \mathrm{mg} / \mathrm{mL}$ Gentamicin (Thermo Fisher Scientific). On day 3, the E8 medium was replaced with one of three treatments, as follows. Stem cell maintenance was continued with serum- and xeno-free E8 medium on vitronectin-coated plates (Thermo Fisher Scientific). The second treatment consisted of "osteogenic medium" containing E8 supplemented with 10\% FBS (Thermo Fisher Scientific), $100 \mu \mathrm{M}$ 2-phospho L-ascorbic acid (Sigma-Aldrich), $10 \mathrm{nM}$ dexamethasone (Sigma-Aldrich), and $5 \mathrm{mM} \mathrm{B-glycerophosphate} \mathrm{(Sigma-Aldrich).}$ The third treatment —osteogenic medium with MET blocking - was as above but also contained $5 \mathrm{nM}$ Capmatinib (Selleckchem, Houston, TX, USA), an ATP-competitive inhibitor of the MET receptor. For all treatments, the media were replaced every 2 days, and the medium and cells were harvested on days 7, 14, and 21.

The cell culture supernatants were analyzed with a Quantikine human HGF ELISA (R\&D systems, Minneapolis, MN, USA). All samples from the three expanded primary cultures (biological replicates) were run as duplicates (technical replicates), in accordance with the manufacturer's instructions, along with the standard curve, and controls. Culture supernatants of the PD-MSCs cultured in osteogenic and osteogenic MET-blocked medium were diluted at a ratio of 1:5 with distilled water before conducting the assay.

The optical density (OD) of each well was determined by using a Synergy 2 multi-mode microplate reader (Biotek, Winooski, VT, USA) at a wavelength of $450 \mathrm{~nm}$ with correction at $540 \mathrm{~nm}$. Corrected raw OD values were interpolated with the linear standard curve, the dilutions were corrected, and the coefficient of variation $(\mathrm{CV})$ was calculated with $\mathrm{Gen} 5$ data analysis software. The percentage change from E8 medium was determined by using the mean value of PD-MSCs cultured in E8 medium against the HGF concentration in osteogenic medium or osteogenic MET-blocked medium at the three time points and paired $t$-test results were calculated by using GraphPad Prism as previously reported (GraphPad Prism version 7.00 for Mac, GraphPad Software, La Jolla, CA, USA; www.graphpad. com) $[13,14]$. The detection limit of the assay was $0.13 \mathrm{ng} / \mathrm{mL}$ of HGF.

\section{Determining the relative expression of osteogenic-related genes}

Cells ( $n=3$, biological repeats) were cultured as outlined above in E8, osteogenic, and osteogenic plus $5 \mathrm{nM}$ Capmatinib media. Total cellular RNA was isolated on days 7, 14, and 21 with a NucleoSpin RNA extraction kit (Macherey-Nagel, Duren, Germany) in accordance with the manufacturer's protocol.

The RNA concentration in each sample was determined using a Nanodrop spectrophotometer. Total RNA (600 ng) was reverse transcribed with a High-Capacity cDNA Reverse Transcription kit (Applied Biosystems, Foster City, CA, USA) in accordance with the manufacturer's instructions.
In addition, five samples were prepared without MultiScribe Reverse Transcriptase as RT negative controls (-RT control).

To quantitatively analyze expression of genes involved in osteogenesis, quantitative reverse transcription real-time polymerase chain reaction (qRT2-PCR) assays were performed using TaqMan chemistry with FAM as the reporter dye. TaqMan Array 96-well fast plates were custom-ordered (Thermo Fisher Scientific) with 12 osteogenesis-related genes of interest -MET, HGF, SRY-Box transcription factor 9 (SOX9), Sp7 transcription factor (SP7), activating transcription factor 4 (ATF4), RUNX family transcription factor 2 (RUNX2), FOS-like 1, AP1 transcription factor subunit (FOSL1), alkaline phosphatase-biomineralization associated (ALPL), bone gamma-carboxyglutamate protein (BGLAP), collagen type I alpha 1 chain (COL1A1), Sp3 transcription factor (SP3), and neural EGFL like 1 (NELL1) - and three housekeeping (reference) genes for normalization: glyceraldehyde-3-phosphate dehydrogenase (GAPDH), beta-2-microglobulin (B2M), and phosphoglycerate kinase 1 (PGK1). Assays $(10-\mu \mathrm{L}$ reactions) were performed on a QuantStudio 6 Real-Time PCR device (Applied Biosystems) with TaqMan Fast Universal PCR Master Mix (Applied Biosystems).

Raw quantification cycle $(\mathrm{Cq})$ values for each reaction were recorded, and expression levels of target genes were normalized against the housekeeping genes. The housekeeping genes GADPH, B2M, and PGK1 were validated by using GeNorm software. Gene expression was calculated with the $2^{-\Delta \Delta \mathrm{Cq}}$ method, and $P$ values (Dunnett's multiple comparison test) were calculated by using the PCR Gene analysis Excel template (SA Bioscience, Frederick, MD, USA) and GraphPad Prism. Genes with a fold regulation greater than \pm 2 and a $P$ of $<0.05$ (Dunnett's multiple comparison test) were considered to be significantly regulated.

\section{Results}

\section{HGF and MET protein expression in human PP}

The HGF immunohistochemical staining pattern in PP tissue was very similar in all three patients (Fig. 1). HGF protein (brown) was detected at moderate levels in the epithelium but not in the outer orthokeratinized stratum corneum layer (Fig. 1A). Within the connective tissue, HGF protein was detected in association with vascular endothelial cells and pericytes of blood vessels (Fig. 1B). HGF protein was also detected in association with cells of the periosteum layer immediately adjacent to the bone (Fig. 1C).

Immunohistochemical staining of PP for the MET protein yielded consistent results for all three samples. Strong positive staining was detected in all epithelial layers except the orthokeratinized stratum corneum layer (Fig. 2A). MET was highly expressed in perivascular cells of capillaries and small blood vessels, which were distributed throughout the PP, as well as the large vessels (Fig. 2A, B). Multiple clusters of MET-positive cells were present in the lamina propria layer near the epithelial rete-pegs, consistent with the distribution of blood vessels (Fig. 2B). Moderate MET expression was detected in cells throughout the osteoblastic layer and in cells adjacent to the bone (Fig. 2C). In contrast, the isotype-matched negative control samples showed only diffuse background staining (Fig. 3A, B).

\section{HGF protein expression in vitro}

HGF protein production was greater for PD-MSCs grown in osteogenic medium than for those grown in the E8 stem cell medium. The standard curve for the HGF ELISA showed a strong linear fit $\left(\mathrm{R}^{2}=1\right)$ with $<8 \%$ $\mathrm{CV}$ for duplicate samples. No HGF protein was detected in the freshly prepared E8 or osteogenic medium. HGF protein production was very low in culture supernatants of undifferentiated PD-MSCs in E8 medium from all three expanded primary cultures (means: $0.25 \mathrm{ng} / \mathrm{mL}$ on day $7,0.68 \mathrm{ng} /$ $\mathrm{mL}$ on day 14 , and $0.42 \mathrm{ng} / \mathrm{mL}$ on day 21 ; limit of detection $=0.13 \mathrm{ng} / \mathrm{mL}$; Table 1). In osteogenic medium, mean HGF concentration increased from $5.37 \mathrm{ng} / \mathrm{mL}$ on day 7 (range: 2.25 to $9.93 \mathrm{ng} / \mathrm{mL}$ ) to 12.52 on day $14 \mathrm{ng} /$ $\mathrm{mL}$ (range: 3.02 to $17.91 \mathrm{ng} / \mathrm{mL}$ ); the level on day $21,12.19 \mathrm{ng} / \mathrm{mL}$, was almost unchanged (range: 2.79 to $18.79 \mathrm{ng} / \mathrm{mL}$ ). In contrast, HGF levels in E8 medium had a range between 0.00 and $1.59 \mathrm{ng} / \mathrm{mL}$ throughout the experiment (Table 1).

When the data were normalized to the mean HGF concentration in control E8 medium at each time point, they showed a normal distribution. There was a significant difference between the control (E8 medium) and osteogenic medium on days $7(P=0.047), 14(P=0.034)$, and $21(P=$ 

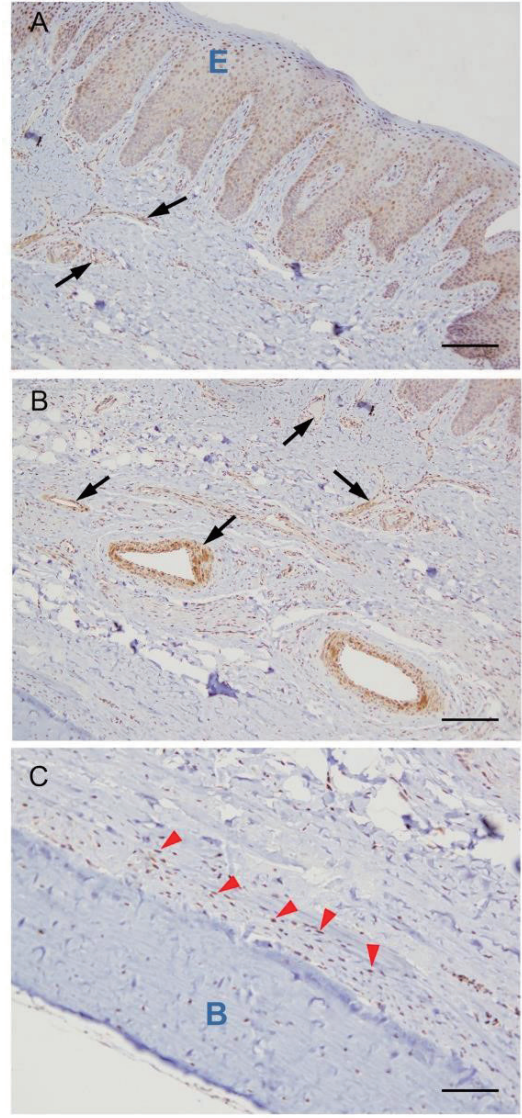

Fig. 1 Representative images of Hepatocyte Growth Factor (HGF) immunohistochemical staining (brown) of human hard palate tissue. (A) Epithelium, lamina propria, submucosa. (B) Submucosa and periosteum. (C) Periosteum and bone. Tissue was counterstained with hematoxylin. E, epithelium; B, bone; Black arrows, blood vessels (example); Red arrowheads, positive cells in ' $\mathrm{C}$ ' (example). Scale bars: A and B, $150 \mu \mathrm{m} ; \mathrm{C}, 50 \mu \mathrm{m}$
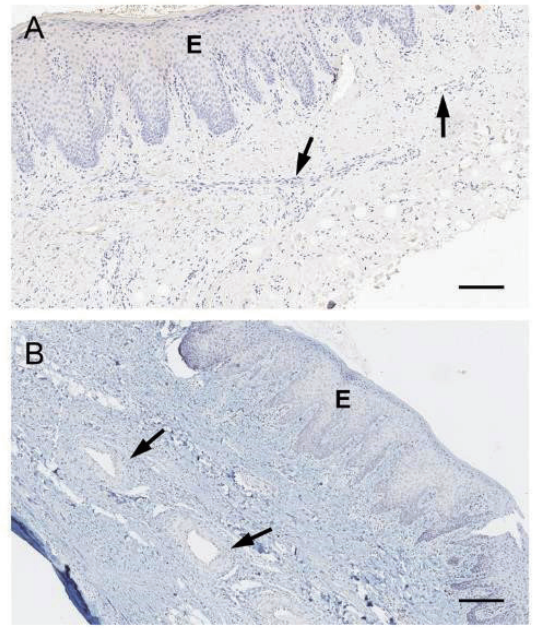

Fig. 3 Representative image of human hard palate with control IgG. (A) Rabbit IgG and (B) mouse IgG. Tissue was counterstained with hematoxylin. E, epithelium; Black arrows, blood vessel (example). Scale bars, $200 \mu \mathrm{m}$

0.036; paired $t$-tests; Fig. 4). The E8 and osteogenic MET-blocked medium significantly differed only on day 7 (day $7, P=0.045$; day $14, P=0.055$; day $21, P=0.064$; paired $t$-tests), even though there was a marked increase in the amount of HGF produced by cells in osteogenic MET-blocked medium (Fig. 4).

\section{Regulation of osteogenic-related genes}

Good quality RNA was extracted: the absorbance A260/A280 ratio was $2.01 \pm 0.06($ mean $\pm \mathrm{SD})$ and the mean $\pm \mathrm{SD}$ yield of eluted RNA was 0.57 $\pm 0.33 \mu \mathrm{g} / \mathrm{mL}$. The TaqMan gene expression detection threshold was set at $\mathrm{Cq}=40$. No genomic DNA was detected $(\mathrm{Cq}>40)$ in the minus reverse
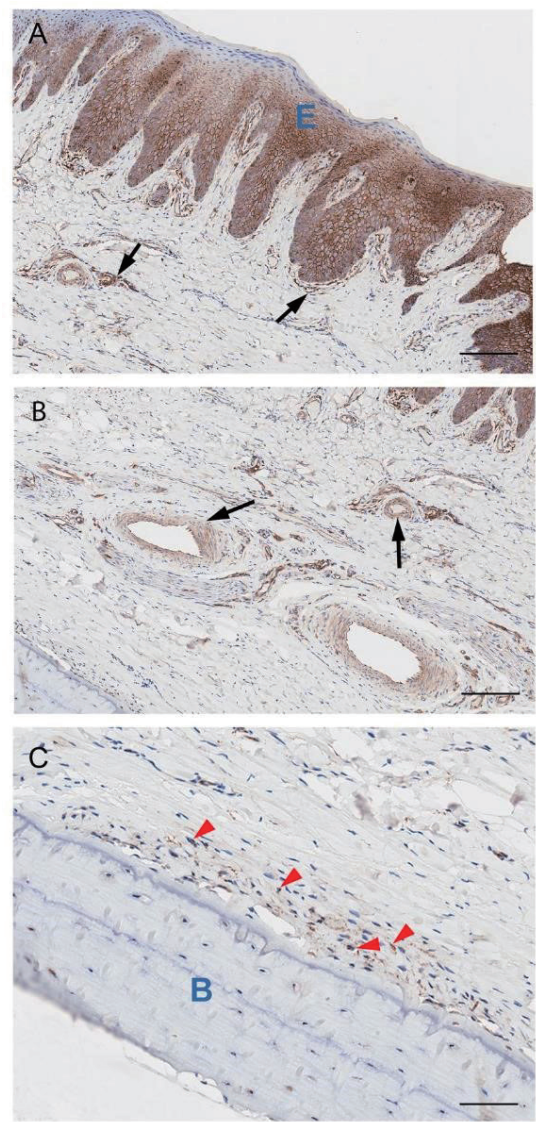

Fig. 2 Representative images of MET proto-oncogene receptor tyrosine kinase (MET) immunohistochemical staining (brown) of human hard palate tissue. (A) Epithelium, lamina propria, submucosa. (B) Submucosa and periosteum. (C) Periosteum and bone. Tissue was counterstained with hematoxylin. E, epithelium; B, bone; Black arrows, blood vessels (example); Red arrowheads, positive cells in 'C' (example). Scale bars: A and B, $150 \mu \mathrm{m}$; C, $50 \mu \mathrm{m}$

Table 1 Hepatocyte Growth Factor (HGF) concentration (ng/mL) in different media

\begin{tabular}{lccc}
\hline & \multicolumn{3}{c}{ Mean HGF concentration $(\mathrm{ng} / \mathrm{mL} \pm \mathrm{SD})$} \\
\hline Essential 8 medium & Osteogenic medium & Osteogenic MET-blocked medium \\
\hline Day 14 & $0.25 \pm 0.27$ & $5.37 \pm 4.05$ & $4.98 \pm 3.67$ \\
Day 21 & $0.68 \pm 0.79$ & $12.52 \pm 8.25$ & $13.87 \pm 11.19$ \\
\hline
\end{tabular}

MET, MET proto-oncogene receptor tyrosine kinase

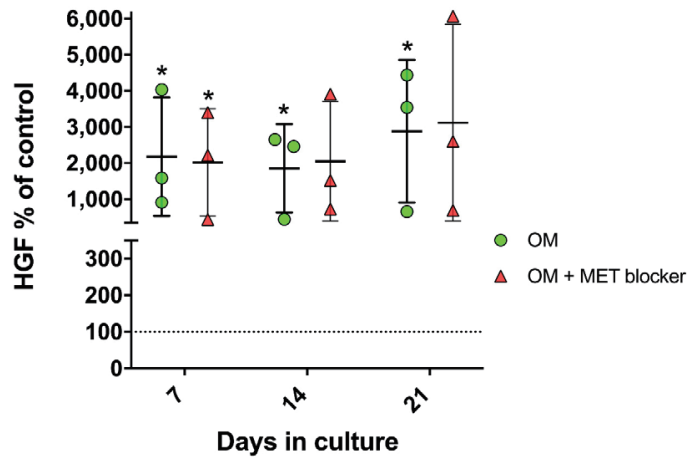

Fig. 4 Hepatocyte growth factor (HGF) protein produced by palatal periosteal-derived mesenchymal stem cells (PD-MSCs), as measured by ELISA. Data presented as percentage of Essential 8 medium (control) at 100\% (dotted line). Media from expanded primary cultures of PD-MSCs were analyzed at days 7, 14, and 21 in osteogenic medium (green circles: OM) and osteogenic MET proto-oncogene receptor tyrosine kinase (MET)-blocked medium (red triangles: OM + MET blocker). ${ }^{*} P \leq 0.05$ as compared with control Essential 8 medium (paired $t$-tests). Mean $\pm \mathrm{SD}$

transcriptase controls (-RT controls) and no signal was detected in the No Template Control samples. The abundance and stability of the housekeeping genes GADPH, B2M, and PGK1 had M-values of $<0.7$ and were thus all used for data normalization. 

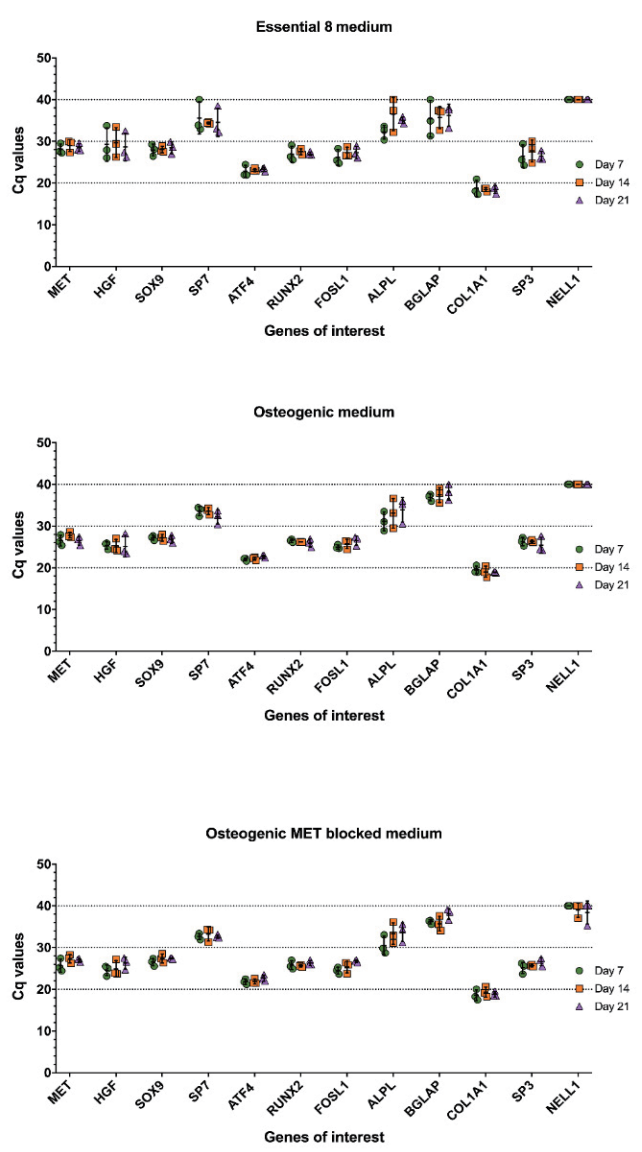

Fig. 5 Quantification cycle $(\mathrm{Cq})$ values for genes of interest from periosteal-derived mesenchymal stem cells (PD-MSCs) cultured in Essential 8 medium, osteogenic medium, and osteogenic MET proto-oncogene receptor tyrosine kinase (MET)-blocked medium at day 7, 14, and 21. Dotted lines $-\mathrm{Cq}=40$ : genes not detected; $\mathrm{Cq}>30$ : low expression; $20<\mathrm{Cq}<30$ : moderate expression; $\mathrm{Cq}<20$ : high expression. Mean $\pm \mathrm{SD}$

Regardless of media, the majority of osteogenic genes investigated were moderately expressed $(20<\mathrm{Cq}<30)$ (Fig. 5). COL1A1 was the most highly expressed gene $(\mathrm{Cq}<20)$, and NELL1, SP7, ALPL, and BGLAP had a $\mathrm{Cq}$ of $>30$, indicating lower levels of RNA.

Among the 12 chosen genes of interest, MET, ATF4, and RUNX2 were significantly downregulated by more than 2 -fold $(P \leq 0.05$, Dunnett's multiple comparison test) on day 21 in osteogenic and osteogenic METblocked medium, as compared with E8, with fold regulations of -2.26 to -6.10 (Fig. 6). There was no significant regulation $(P>0.05$ and fold regulation between \pm 2 ) of genes when osteogenic cell cultures with and without MET blocking were compared. The RUNX2 gene was significant ( $P=0.0012$, Dunnett's multiple comparison test) with MET blocking but had a fold upregulation of only 1.67. HGF gene expression levels were not significantly regulated by culture conditions, and large variations in expression levels were evident (Fig. 6).

Expressions of SOX9, SP7, FOSL1, ALPL, SP3, COL1A1, and BGLAP genes were not regulated at any timepoint; however, E8 medium yielded the greatest variability in expression, with tighter regulation seen when cells were cultured in osteogenic conditions (Fig. 7). NELL1 was only expressed at low levels in one out of three PD-MSCs exposed to osteogenic MET-blocked medium; however, this was noted at both day 14 and day 21 (Fig. 7).

\section{Discussion}

The remarkable role of periosteum in bone healing and regeneration and the maintenance of bone homeostasis has long been recognized. The osteogenic properties of PD-MSCs were successfully used as an alternative or adjunct to bone grafts and osteoconductive scaffolds when treating critical-sized bone defects $[15,16]$. BMP2, the only commercially available FDA approved growth factor to enhance osteogenesis, has been used for this purpose; however, high costs, biological instability, and the lack
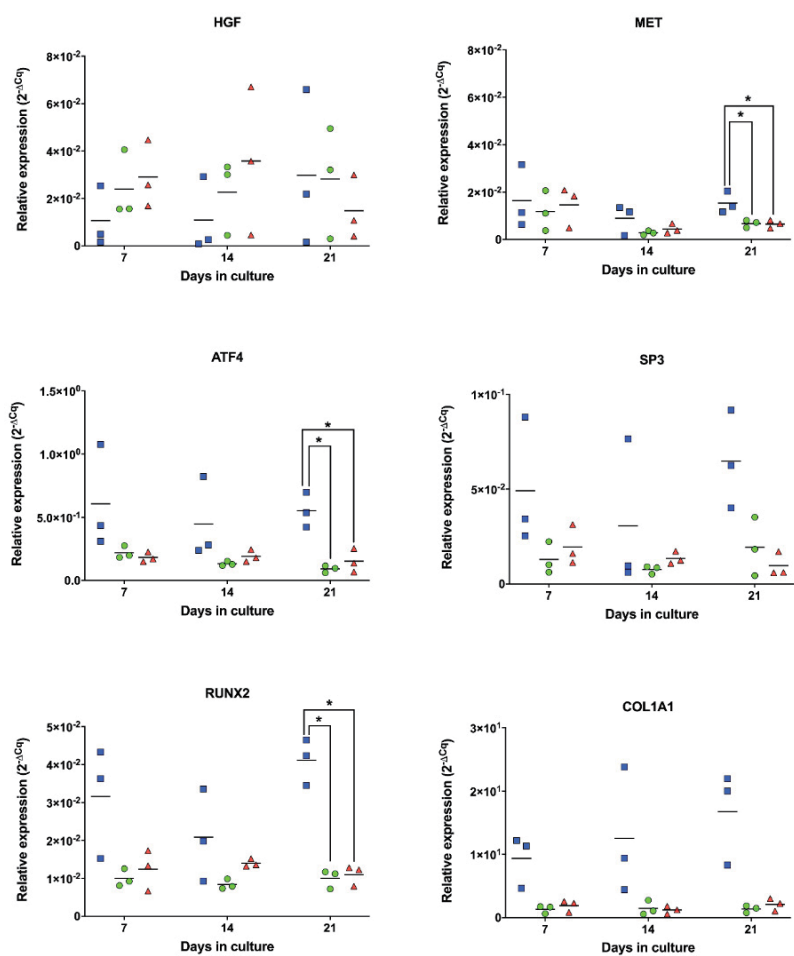

Fig. 6 Relative expression $\left(2^{-\Delta C \mathrm{C}}\right)$ of osteogenic-related genes (HGF, MET, ATF4, SP3, RUNX2, COL1A1) at days 7, 14, and 21 from cultured palatal periosteal-derived mesenchymal stem cells (PD-MSCs). Essential 8 stem cell medium (blue squares), osteogenic medium (green circles), or osteogenic MET-blocked medium (red triangles). (- = mean), ${ }^{*} P \leq 0.05$ (Dunnett's multiple comparison test)
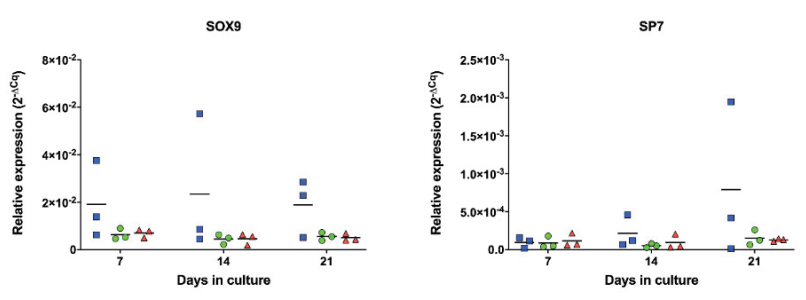

FosL

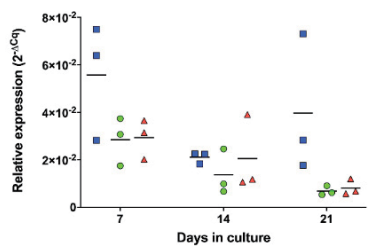

ALPL
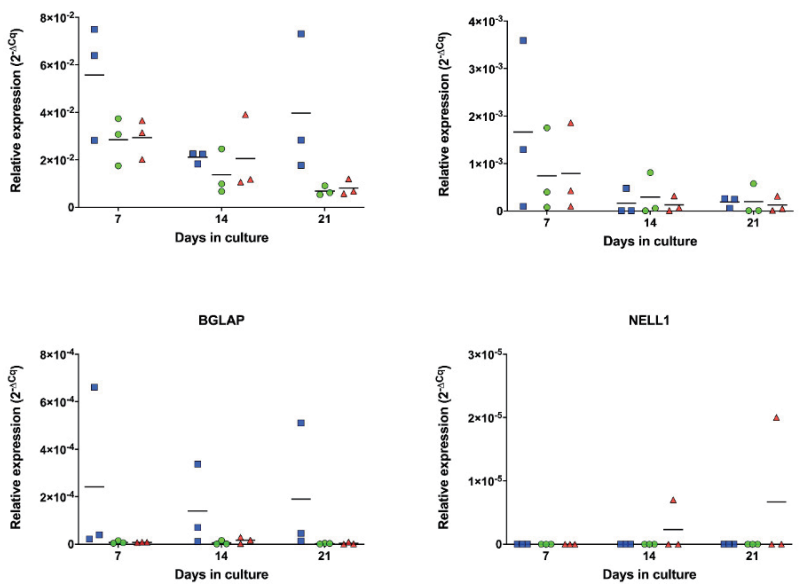

Fig. 7 Relative expression $\left(2^{-\Delta C a}\right)$ of osteogenic-related genes (SOX9, SP7, FOSL1, ALPL, BGLAP, NELL1) at days 7, 14, and 21 from palatal periosteal-derived mesenchymal stem cells (PD-MSCs) cultured in three different media. Essential 8 stem cell medium (blue squares), osteogenic medium (green circles), or osteogenic MET proto-oncogene receptor tyrosine kinase (MET)-blocked medium (red triangles). (- = mean), ${ }^{*} P \leq 0.05$ (Dunnett's multiple comparison test)

of long-term risk assessment studies limit its widespread clinical use [17]. Identifying alternative effective inducers of osteogenesis, such as PDMSCs, and determining whether HGF/MET might play an important role in osteogenesis are interesting avenues for future therapies.

This study investigated the location of HGF and MET-positive cells 
in human PP and quantified the concentration of HGF produced by PDMSCs during osteogenesis in vitro. Blocking of the MET receptor during osteogenesis in vitro was investigated in conjunction with expressions of key osteogenic genes in E8 and osteogenic media. Within human PP, the MET receptor was detected in perivascular cells of small and large blood vessels and in the epithelium. In addition, the fibrous layer of the periosteum near the bone contained numerous MET-positive cells. HGF protein was also localized to blood vessels and epithelium and proximal to bone. In the cultured PD-MSCs, moderate levels of MET and HGF mRNA transcripts were detected, supporting a possible autocrine MET/HGF signaling pathway during osteogenesis. Thus, in addition to the typical paracrine epithelial-mesenchymal signaling mechanism, the HGF/MET complex within the PP may utilize an autocrine activation loop. The location of HGF/MET suggests an angiogenic role in addition to the regulation of MSCs known to be present in the perivascular space.

HGF/MET signaling was reported to be important in regulating organogenesis, tissue repair, and wound healing, as well as osteogenesis [18]. Growing evidence indicates that HGF increases expression of key osteogenic markers and improves osteogenesis via the BMP-2-mediated signaling pathway [19]. Conversely, inhibition of the HGF receptor MET enhanced BMP-2-induced osteoblast differentiation [6]. The HGF/MET pathway has been extensively studied, and mutation and overexpression of the MET protein lead to cancer development and are associated with poor prognosis; however, few studies have examined the physiological role of HGF/MET during osteogenesis [20].

The MET receptor tyrosine kinase and its only known ligand, HGF, are important in controlling tissue homeostasis via multiple biological functions and regulate a range of cellular signaling pathways involved in cell viability, motility, proliferation, migration, and invasion [21]. Recent evidence suggests that MSCs and osteoblasts may express MET and HGF and that this signaling pathway has a role in osteogenesis. This raises the possibility that HGF/MET has a role in human PP maintenance and that PD-MSCs are a potential source of osteogenic induction [1].

E8 medium, in which FBS is substituted with seven essential nutrients, was used to select for a MSC population in this study [9]. Any clinical translation requiring amplification of PD-MSCs should consider using serum- and xeno-free media, to reduce the risk of disease transmission and batch-to-batch variability found with animal serum. The present authors' previous FACS analysis revealed that these same cultured PD-MSCs contained CD73 (99.9-100\%) and CD90 (98.9-99.9\%). In addition, most cells also expressed CD105 (66.9-80.2\%). Further analysis revealed that $99.5 \%$ of all live single cells co-expressed the MSC cell-surface antigens CD90 and $\mathrm{CD} 73 ; 73.0 \%$ of these double-positive populations were also positive for CD105. Only $0.5 \pm 0.3 \%$ (mean $\pm \mathrm{SD}$ ) were positive for hematopoietic stem cell markers (CD45, CD34, CD11b, CD19, HLA-DR) [9]. Lower CD105 positivity may be related to technical issues during collection, culture, or analysis; however, other groups have reported that stem cells cultured in serum-free medium express lower levels of CD105 while maintaining expression of CD73 and CD90 [22,23]. The addition of $10 \%$ FBS and triple supplement (L-ascorbic acid, dexamethasone B-glycerophosphate) to the E8 medium was previously reported to induce osteogenesis in these cells, as measured by nodule formation, von Kossa staining, and Alizarin Red S and osteocalcin immunostaining [9]. Phenotypically, the cells thus undergo osteogenesis when induced.

In this study, mean HGF concentrations in osteogenic and osteogenic MET-blocked medium were 5.37 and $4.98 \mathrm{ng} / \mathrm{mL}$ at day $7,12.52$ and $13.87 \mathrm{ng} / \mathrm{mL}$ at day 14 , and 12.19 and $13.21 \mathrm{ng} / \mathrm{mL}$ at day 21 , a significant increase as compared with cells kept in stem cell medium alone (mean levels: $0.25-0.68 \mathrm{ng} / \mathrm{mL}$ ). The significant increase in HGF produced by the PD-MSCs undergoing osteogenesis suggests HGF is important in the induction or control of this process. This study was not designed to test for the effects of FBS on HGF production, although a previous study found that FBS may stimulate HGF secretion [10]. However, other studies reported that serum starvation increases the production of HGF, as a protective factor for stressed cells $[11,24]$. A feedback loop between receptor regulation and ligand production was not found. Interestingly, even though HGF protein levels were higher in osteogenic medium, gene expression levels of HGF were not different, and HGF gene expression was highly variable. This suggests that translational regulation of HGF or changes in the half-life of the RNA may be of greater significance during regulation of HGF production.

HGF induces cell scattering in epithelium and epithelial cancer cell lines [25]. In addition, an HGF-related cell scattering effect was observed in cells during serum starvation and the associated cellular stress [11]. Increased expression of HGF protein during osteogenesis has not been directly associated with cell scattering and may have functions involving distinctive pathways and regulators in response to different biological needs.

In this study, cultured unstimulated PD-MSCs in E8 medium expressed all the investigated osteogenic-related genes except NELL1. This suggests that these PP-derived cells, which were previously shown to be MSCs capable of trilineage differentiation, may have a phenotype conducive to becoming bone. Others have also reported that culture of stem cells from dental pulp in serum-free media results in greater upregulation of multiple genes, which included osteogenic genes, as compared with serum-containing media, and that E8 medium results in greater expression of osteogenic-associated genes [26,27]. This confirms the observation that culturing oral MSCs in serum-free media results in an "osteogenic predisposition" [28]

RUNX2 and SP7 (Osterix), which are central osteoblastic and maturation control regulator genes [29], were expressed alongside the ATF4 gene, which is responsible for inducing expression of these key regulatory genes. FOSL1, which is a subunit of the AP-1 complex and important in regulating bone matrix formation, was also produced [30]. PD-MSCs also expressed ALPL and COL1A1, which are early osteoblastic differentiation indicator genes, and SP3 and BGLAP (osteocalcin), which are responsible for maturation of osteoblasts and production of mineralized protein [31]. SOX9 is normally expressed by osteochondroprogenitor cells and chondrocytes, and its expression by PD-MSCs suggests that periosteal-derived MSCs could be utilized clinically to repair and regenerate bone and cartilage.

Collagen type I mRNA was the most abundantly expressed gene at all time points. This is consistent with the in vivo milieu, in which the outer periosteum is predominantly composed of collagen to help it withstand functional mechanical stresses [32]. Because approximately $90 \%$ of total mineralized bone matrix proteins are collagen 1 , the abundant COL1A1 gene expression by unstimulated PD-MSCs in this study could make them a viable stem cell source for bone engineering applications. The second most abundantly expressed gene in this study was ATF4, which is a key regulator of RUNX2 and SP3 expression and is required for coordinating osteogenesis [33].

The expression of all the osteogenic genes of interest by the osteogenically induced cells was comparable to the expression levels of unstimulated PD-MSCs at all time points (days 7, 14, and 21), except for MET, ATF4, and RUNX2, which were significantly downregulated during the final matrix mineralization and maturation stage on day 21 . A possible explanation for this is that the osteogenically differentiated cells had undergone transformation into mature osteoblasts, which decreased the relative gene expression levels of key early osteogenic regulator genes (RUNX2, ATF4). Likewise, the role of the MET gene might be more important during early osteogenesis than during bone nodule maturation.

Expression levels did not significantly differ between PD-MSCs cultured in OM and MET-blocked OM. Capmatinib, the most potent (on the basis of its IC50) and highly selective inhibitor of MET, was used in this study. However, no significant difference was observed in the relative expressions of MET and HGF, or any other osteogenic genes of interest except, possibly, SP3 and NELL1. The essential transcription factor required for maturation of osteoblasts, SP3, was significantly downregulated under MET-blocked OM, as compared with unstimulated cells. Zhang et al. (2003) discovered an important role for SP3 in the regulation of MET gene in different types of renal cells [34]. Similarly, NELL1 was not expressed at any timepoint by unstimulated or osteogenic differentiated PD-MSCs in the present study. However, on days 14 and 21, one of the three tested MET-blocked cultures had higher levels of NELL1 expression on day 14, and a peak level on day 21. The osteoinductive factor NELL1 was reported to promote bone regeneration during rat femoral distraction osteogenesis and in murine cavarial osteolysis models [35]. In addition, NELL1 protein is responsible for premature fusion of sutures in craniosynostosis [36]. Therefore, future studies with larger samples should attempt to clarify the relationship between NELL1 and MET in the context of osteogenesis.

In summary, the importance of HGF/MET in human PP and osteogen- 
esis was investigated. In vivo immunohistochemistry detected high levels of co-localized HGF and MET protein. The PP tissue was harvested and cultured as MSCs in E8 medium, which was then compared with cells induced to undergo osteogenesis. HGF levels were significantly higher in the osteogenic medium than in the MSC medium at days 7, 14, and 21. The gene expression profile suggests that the PD-MSC were expressing osteogenic genes at levels comparable to those in osteogenically induced cells. The study highlights the potential uses of serum-free cultured PD-MSCs for regenerative medicine and the role of HGF/MET in PP maintenance.

\section{Acknowledgments}

The authors thanks Prof. Stephen Robertson and Dr. Christine Neyt for collaboration, suggestions, advice, and support for this project and Dr. Trudy Milne for technical assistance with qPCR.

\section{Conflict of interest}

The authors declare no conflicts of interest.

\section{References}

1. Neuss S, Becher E, Wöltje M, Tietze L, Jahnen-Dechent W (2004) Functional expression of HGF and HGF receptor/c-met in adult human mesenchymal stem cells suggests a role in cell mobilization, tissue repair, and wound healing. Stem Cells 22, 405-414.

2. Frisch RN, Curtis KM, Aenlle KK, Howard GA (2016) Hepatocyte growth factor and alternative splice variants - expression, regulation and implications in osteogenesis and bone health and repair. Expert Opin Ther Targets 20, 1087-1098.

3. Harshman LC, Choueiri TK (2013) Targeting the hepatocyte growth factor/c-Met signaling pathway in renal cell carcinoma. Cancer J 19, 316-323.

4. Naldini L, Weidner KM, Vigna E, Gaudino G, Bardelli A, Ponzetto C et al. (1991) Scatter factor and hepatocyte growth factor are indistinguishable ligands for the MET receptor. EMBO J 10, 2867-2878.

5. Wen Q, Zhou L, Zhou C, Zhou M, Luo W, Ma L (2012) Change in hepatocyte growth factor concentration promote mesenchymal stem cell-mediated osteogenic regeneration. J Cell Mol Med 16, 1260-1273.

6. Kim JW, Lee MN, Jeong BC, Oh SH, Kook MS, Koh JT (2017) Chemical inhibitors of c-Met receptor tyrosine kinase stimulate osteoblast differentiation and bone regeneration. Eur J Pharmacol 806, 10-17

7. Gray MJ, Kannu P, Sharma S, Neyt C, Zhang D, Paria N et al. (2015) Mutations preventing regulated exon skipping in MET cause osteofibrous dysplasia. Am J Hum Genet 97, 837847.

8. Wang Y, Han ZB, Song YP, Han ZC (2012) Safety of mesenchymal stem cells for clinical application. Stem Cells Int 2012, 652034.

9. Naung NY, Duncan W, De Silva R, Coates D (2019) Localization and characterization of human palatal periosteum stem cells in serum-free, xeno-free medium for clinical use. Eur J Oral Sci 127, 99-111.

10. Ohshima M, Yokosuka R, Yamazaki Y, Tokunaga T, Maeno M, Otsuka K (2002) Effects of serum on hepatocyte growth factor secretion and activation by periodontal ligament and gingival fibroblasts. J Periodontol 73, 473-478.

11. Jun EJ, Kim HS, Kim YH (2007) Role of HGF/c-Met in serum-starved ARPE-19 cells. Korean J Ophthalmol 21, 244-250.

12. Liu X, Wang Q, Yang G, Marando C, Koblish HK, Hall LM et al. (2011) A novel kinase inhibitor, INCB28060, blocks c-MET-dependent signaling, neoplastic activities, and crosstalk with EGFR and HER-3. Clin Cancer Res 17, 7127-7138.

13. Zafar S, Cullinan MP, Drummond BK, Seymour GJ, Coates DE (2020) Effects of zoledronic acid and geranylgeraniol on angiogenic gene expression in primary human osteoclasts. J Oral Sci $62,79-83$
14. Porter GC, Duncan WJ, Jude A, Abdelmoneim D, Easingwood RA, Coates DE (2021) Endocytosed silver nanoparticles degrade in lysosomes to form secondary nanoparticle structures during expression of autophagy genes in osteogenic cells. Nanomedicine 33 , 102355.

15. Nagata M, Hoshina H, Li M, Arasawa M, Uematsu K, Ogawa S et al. (2012) A clinical study of alveolar bone tissue engineering with cultured autogenous periosteal cells: coordinated activation of bone formation and resorption. Bone 50, 1123-1129.

16. Yousefi AM, James PF, Akbarzadeh R, Subramanian A, Flavin C, Oudadesse H (2016) Prospect of stem cells in bone tissue engineering: a review. Stem Cells Int 2016, 6180487.

17. Herford AS (2017) The use of recombinant human bone morphogenetic protein-2 (rhBMP2) in maxillofacial trauma. Chin J Traumatol 20, 1-3.

18. Nakamura T, Sakai K, Nakamura T, Matsumoto K (2011) Hepatocyte growth factor twenty years on: much more than a growth factor. J Gastroenterol Hepatol 26, 188-202.

19. Zhen R, Yang J, Wang Y, Li Y, Chen B, Song Y et al. (2018) Hepatocyte growth factor improves bone regeneration via the bone morphogenetic protein 2 mediated NFkappaB signaling pathway. Mol Med Report 17, 6045-6053.

20. Mo HN, Liu P (2017) Targeting MET in cancer therapy. Chronic Dis Transl Med 3, 148153 .

21. Bussolino F, Di Renzo MF, Ziche M, Bocchietto E, Olivero M, Naldini L et al. (1992) Hepatocyte growth factor is a potent angiogenic factor which stimulates endothelial cell motility and growth. J Cell Biol 119, 629-641.

22. Alexander D, Rieger M, Klein C, Ardjomandi N, Reinert S (2013) Selection of osteoprogenitors from the jaw periosteum by a specific animal-free culture medium. PLoS One 8, e81674.

23. Mark P, Kleinsorge M, Gaebel R, Lux CA, Toelk A, Pittermann E et al. (2013) Human mesenchymal stem cells display reduced expression of CD105 after culture in serum-free medium. Stem Cells Int 2013, 698076

24. Nakamura T, Mizuno S (2010) The discovery of hepatocyte growth factor (HGF) and its significance for cell biology, life sciences and clinical medicine. Proc Jpn Acad Ser B Phys Biol Sci 86, 588-610

25. Grotegut S, von Schweinitz D, Christofori G, Lehembre F (2006) Hepatocyte growth factor induces cell scattering through MAPK/Egr-1-mediated upregulation of Snail. EMBO J 25, $3534-3545$

26. Harada K, Kawai S, Wen-an X, liang X, Sonomoto M, Shinonaga Y et al. (2015) Alterations in deciduous dental pulp cells cultured with serum-free medium. J Hard Tissue Biol $24,17-22$.

27. Xiao J, Yang D, Li Q, Tian W, Guo W (2018) The establishment of a chemically defined serum-free culture system for human dental pulp stem cells. Stem Cell Res Ther 9, 191.

28. Bakopoulou A, Apatzidou D, Aggelidou E, Gousopoulou E, Leyhausen G, Volk J et al. (2017) Isolation and prolonged expansion of oral mesenchymal stem cells under clinicalgrade, GMP-compliant conditions differentially affects "stemness" properties. Stem Cell Res Ther 8, 247.

29. Jensen ED, Gopalakrishnan R, Westendorf JJ (2010) Regulation of gene expression in osteoblasts. Biofactors 36, 25-32

30. Eferl R, Hoebertz A, Schilling AF, Rath M, Karreth F, Kenner L et al. (2004) The Fosrelated antigen Fra-1 is an activator of bone matrix formation. EMBO J 23, 2789-2799.

31. Brennan-Speranza TC, Conigrave AD (2015) Osteocalcin: an osteoblast-derived polypeptide hormone that modulates whole body energy metabolism. Calcif Tissue Int 96, 1-10.

32. Ferretti C, Mattioli-Belmonte M (2014) Periosteum derived stem cells for regenerative medicine proposals: Boosting current knowledge. World J Stem Cells 6, 266-277.

33. Yang X, Karsenty G (2004) ATF4, the osteoblast accumulation of which is determined post-translationally, can induce osteoblast-specific gene expression in non-osteoblastic cells. J Biol Chem 279, 47109-47114.

34. Zhang X, Li Y, Dai C, Yang J, Mundel P, Liu Y (2003) Sp1 and Sp3 transcription factors synergistically regulate HGF receptor gene expression in kidney. Am J Physiol Renal Physiol 284, F82-94.

35. Guo X, Peng J, Wang Y, Wang A, Zhang X, Yuan M et al. (2012) NELL1 promotes bone regeneration in polyethylene particle-induced osteolysis. Tissue Eng Part A 18, 1344-1351.

36. Ting K, Vastardis H, Mulliken JB, Soo C, Tieu A, Do H et al. (1999) Human NELL-1 expressed in unilateral coronal synostosis. J Bone Miner Res 14, 80-89. 